\title{
The Value and Analysis of Combined Detection of Tumor Markers in the Diagnosis and Treatment of Lung Cancer
}

\author{
Yiming Li \\ Isotope Laboratory of the Second Affiliated Hospital of Qiqihar Medical University, Heilongjiang Qiqihar \\ 161000, China
}

Keywords: value, analysis, combined detection, tumor markers

\begin{abstract}
Objective: To study the relationship between pathological types of lung cancer and tumor markers and the combined detection of multiple tumor markers for the diagnosis of lung cancer. Methods: The clinical data of 68 patients with lung cancer in this hospital were retrospectively analyzed. The relationship between pathological types of lung cancer and tumor markers was studied. The methodological index was used to evaluate the clinical application value of combined detection of multiple tumor markers in the diagnosis of lung cancer. Results: The serum levels and abnormalities of CEA, CA125 and NSE in lung cancer group were significantly different from those in benign lung lesions and control group $(\mathrm{P}<0.05)$. CEA and CA125 had certain specificity for adenocarcinoma. (68. $5 \pm 21.5) \mu \mathrm{g} \cdot \mathrm{L}-1$, (98. $5 \pm 31.2) \mathrm{U} \cdot \mathrm{mL}-1$ was statistically different from squamous cell carcinoma and small cell carcinoma $(\mathrm{P}<0.05)$. The NSE serum concentration of small cell carcinoma was significantly higher $(45.5 \pm 7.7) \mu \mathrm{g} \bullet \mathrm{L}-1$ compared with squamous cell carcinoma and adenocarcinoma ( $<<0.05)$; patients with stage I to II in the lung cancer group The serum concentrations of CEA, CA125 and NSE were lower than those of stage III-IV. There were significant differences between the two groups $(\mathrm{P}<0.05)$. The sensitivity of the three tumor markers was CEA $51.2 \%$ is better, combined detection can greatly improve the sensitivity of detection (73. 5\%) and the Yoden index (0.57). Conclusion: CEA, CA125 and NSE has important clinical value in the diagnosis, treatment and lung cancer recurrence monitoring. The combined detection of three tumor markers can improve the accuracy of lung cancer diagnosis.
\end{abstract}

\section{Introduction}

Lung cancer is a common malignant tumor that threatens human health. In recent years, its incidence has been increasing year by year, and it has become one of the common diseases that threaten human health. In the past, its diagnosis was mainly based on cytology and histopathology. The specificity was high, but the sensitivity was not high. There are still some drawbacks in the diagnosis of the disease. With the detection of serum tumor markers in the diagnosis and treatment of lung cancer, it is currently the main method for diagnosis and treatment of clinicians, but there is still a problem of sensitivity and specificity in individual tests. Most detection sensitivity is $<40 \%$. Therefore, joint detection is of great significance for the diagnosis and treatment of lung cancer.

The initial symptoms of lung cancer are not obvious, and the blood supply in the lungs is prone to metastasis. It is reported that $70 \%$ to $86 \%$ of lung cancers are in advanced stage when they are found, and the enzyme system mutation and tumor cell synthesis and secretion of embryos in the early stage of cancer Abnormal protein levels are increasingly used in the early diagnosis of cancer in the field of clinical testing. Tumor markers have a certain sensitivity for early diagnosis of cancer. We retrospectively analyzed the clinical data of 68 patients with lung cancer in our hospital, and compared them with tumor markers of patients with benign lung disease and normal physical examination to study lung cancer.

\section{Research Objects and Methods}

68 patients with lung cancer were from January 2010 to November 2012. The inpatients in the oncology department of our hospital were set as lung cancer group. All were diagnosed by 
pathological examination, microfibrous biopsy, CT and surgery, including 39 males and 29 females. For example, age 45 to 68 years, mean (53. $6 \pm 5$. 2) years, 33 cases of squamous cell carcinoma, 25 cases of adenocarcinoma, 10 cases of small cell carcinoma, according to the International Association for the Study of Lung Cancer (IASLC) TNM staging criteria, Phase Ia In 8 cases, there were 13 cases in stage Ib, 14 cases in stage IIa, 11 cases in stage IIb, 10 cases in stage IIIa, 6 cases in stage IIIa, and 6 cases in stage IV. 107 patients with benign lung lesions were selected as benign lesions, including male. There were 63 cases, 44 females, aged 40-70 years, mean (56.1 \pm 5.8$)$ years old, 60 cases of pneumonia, 18 cases of lung abscess, 15 cases of tuberculosis, 12 cases of bronchiectasis, 2 cases of inflammatory pseudotumor; 72 healthy subjects were included in the normal control group, 42 males and 30 females, aged 43-65 years, mean (55.4 \pm 5 . 3) years old. There was no significant difference in gender composition and average age between the groups.

Three groups of tumor markers: carcinoembryonic antigen (CEA), carbohydrate antigen 125 (CA125), neuron enolase (NSE) serum levels and abnormal rates, compare different pathological types of lung cancer and different stages of lung cancer CEA, CA125, The detection of NSE, and the use of methodological indicators to evaluate the sensitivity, specificity, coincidence rate, correct diagnostic index, negative predictive value, and positive predictive value of the three tumor markers alone and in combination.

Three groups of subjects took $5 \mathrm{~mL}$ of venous blood in the morning on an empty stomach, and the serum was separated by centrifugation. The CEA, CA125 and NSE were detected by electrochemiluminescence. The instrument was Roche COBAS6000. The normal reagent range was used: CEA $0 \sim 5.0 \mu \mathrm{g} \bullet \mathrm{L}-1$, CA125 0 to $35 \mathrm{U} \cdot \mathrm{mL}-1$, NSE0 to $20 \mu \mathrm{g} \bullet \mathrm{L}-1$.

Use statistical software SPSS17. 0 The study data were analyzed and processed. The mean between groups was expressed as $\mathrm{x} \pm \mathrm{s}$. The comparison was performed by one-way analysis of variance. The SNK method was used for comparison between groups. The t test was used to compare the mean values. The chi-square test was used to compare the composition ratio and rate.

\section{Related Research on Tumor Markers of Lung Cancer}

Lung cancer is the most common malignant tumor in the clinic and the most common malignant tumor. According to the World Health Organization, lung cancer accounts for about $19 \%$ of all malignant tumors, ranking first in the world for cancer deaths, and its incidence is increasing year by year. Most studies have pointed out that the low rate of early diagnosis is the main reason for the decline in the survival rate of lung cancer patients. The diagnosis rate of stage I lung cancer is about $8 \%$. In clinical practice, more than $50 \%$ of patients are in the middle and late stage when they are diagnosed with lung cancer. This seriously restricts the survival rate of patients, so early diagnosis is particularly important for the treatment of lung cancer and improving the survival rate of patients. In recent years, with the development of molecular biology technology, the value of tumor markers for early detection and early diagnosis of lung cancer has received extensive attention. CEA, NSE, CYFRA21-1, CA125, etc. are clinically used lung cancer tumor markers. The diagnosis of early lung cancer is mostly based on single detection method. Although it has certain value for the diagnosis and prognosis of lung cancer, it still has accuracy. The rate is not high and so on. At present, clinically, the method of combined detection of serum tumor markers is used to assist in the diagnosis of lung cancer, and the research proves that judging the tumor marker level is also beneficial to its prognosis and treatment effect.

CEA is an adhesion molecule in the tumor antigen immunoglobulin superfamily. It exists in the embryonic tissue of the human body. It has immunosuppressive effect and can effectively promote the metastasis of tumor cells. It belongs to a kind of lung cancer cells and can be directly produced. The marker; NSE is the most specific and sensitive tumor marker for small cell lung cancer; CYFRA21-1 is a filamentous substance distributed in the monolayer epithelial cytoskeleton and is a novel tumor marker; CA125 Exist in the body cavity epithelium of the fetus, it is a cell surface high molecular glycoprotein. CA125 detection is effective in the diagnosis, clinical treatment and prognosis of lung cancer. In this study, we can see that the levels of serum tumor markers CEA, 
NSE, CYFRA21-1, and CA125 in lung cancer patients are significantly higher than those in the control group and healthy group $(\mathrm{P}<0.05)$, indicating that CEA, NSE, CYFRA21-1, CEA, NSE, CYFRA21-1, The level of CA125 can promote the early clinical diagnosis of lung cancer patients and has high application value. From the single-sentence and combined detection and diagnosis of tumor markers in Table 2, it can be seen that the accuracy, specificity and sensitivity of lung cancer diagnosis are only low in the accuracy and sensitivity of lung cancer diagnosis by single tumor markers; The accuracy of serum tumor markers for the diagnosis of lung cancer is $86.66 \%$, the specificity is $90.0 \%$, and the sensitivity is $87.5 \%$. This indicates that the combined detection of serum tumor markers for lung cancer is more accurate and sensitive than single detection. The lack of single detection can better assist the early diagnosis of lung cancer, and has certain value for the diagnosis and prognosis of lung cancer.

\section{Discussion}

Tumor markers (TM) are substances that are expressed or secreted during tumor malignant tumorigenesis and proliferation, or are abnormally produced or elevated by the body's response to tumors, reflecting the presence and growth of tumors. It exists in the cells or tissues of patients and can be determined by biochemical, molecular and molecular biological methods. It has clinical value for the auxiliary diagnosis, differential diagnosis, observation of curative effect, monitoring of recurrence and prognosis of tumors. However, the sensitivity and specificity of the currently used tumor markers are limited, and the positive rate of the early stage of the tumor (such as stage I and II) is low, and no "gold standard" tumor markers have been found so far. Moreover, some tumor cells can produce a variety of markers, and a single tumor marker is difficult to accurately reflect the complexity of the tumor, and the combined detection of tumor markers is very clinically useful for tumor diagnosis.

Chen Jing et al reported the clinical value of serum tumor marker detection as follows: squamous cell carcinoma antigen (SCCA): tumor-associated antigen TA-4 extracted from cervical squamous cell carcinoma tissue, normal human serum content Micro $<2.5 \mu \mathrm{g} / \mathrm{L}$. SCCA is a tumor marker for squamous cell carcinoma. Carcinoembryonic antigen (CEA): Carcinoembryonic antigen is a glycoprotein embryonic antigen found in fetal and colon cancer tissues and is a broad-spectrum tumor marker. Serum CEA normal reference value $<5 \mu$ g / L. Pulmonary disease also has varying degrees of elevated CEA levels, but the degree of elevation and positive rate are lower. CEA belongs to adhesion molecules and is an important marker for a variety of tumor metastasis recurrence. CYFRA21-1 is a subunit of epithelial cell structural proteins that are commonly distributed on the surface of normal tissues. In malignant cells, activated proteases accelerate cell degradation and a large amount of cytokeratin fragments are released into the blood. The content of CYFRA21-1 is very abundant in lung cancer tissues, especially in lung squamous cell carcinoma. Carbohydrate antigen 125 (CA125) was originally a glycoprotein detected from epithelial ovarian cancer antigen and belongs to tumor-associated antigen. It is mainly used for diagnosis in lung cancer, especially for the diagnosis of adenocarcinoma. Neuron-specific enolase (NSE): NSE is an isoenzyme of enolase. NSE is a tumor marker for small cell lung cancer (SCLC) with a diagnostic positive rate of $91 \%$. The above tumor markers have different sensitivities to lung cancer, and our results also show that the sensitivity of single detection of each tumor marker to lung cancer is relatively low, and our test results are consistent with the literature reports. The accuracy of each tumor marker alone is limited, and combined detection will increase its sensitivity, specificity and accuracy.

With the rapid development of molecular biology in recent years, many scholars have reported on the detection and rational application of tumor markers, especially in the diagnosis, treatment and follow-up of lung cancer, which has received more and more attention from clinicians. The diagnostic value of five serum tumor markers such as SCCA, CEA, CYFRA21-1, CA125 and NSE for lung cancer has been reported in many literatures. The statistical analysis of this study showed that SCCA and CYFRA21-1 have higher diagnostic value for lung squamous cell carcinoma. CEA and CA125 have higher diagnostic value for lung adenocarcinoma, while NSE has higher sensitivity 
and specificity for small cell lung cancer. It has been reported in the literature [10] that the combined detection of NSE, Cy-fra21-1, CA199, CAl25, CEA five cancer markers for the diagnosis sensitivity, accuracy and specificity of lung cancer are $92.0 \%, 89.0 \%, 88.0 \%$ were significantly better than single tumor markers, and the differences were statistically significant $(\mathrm{P}<0.05)$. There are also reports combined detection of CYFRA21-1, CEA and NSE can significantly improve the sensitivity and accuracy of lung cancer diagnosis, reaching $85.2 \%$ and $84.6 \%$, respectively, compared with any single item. Both were statistically significant. This study showed that the sensitivity, specificity and accuracy of combined detection of SCCA, CEA, CYFRA21-1, CA125 and NSE for lung cancer diagnosis were 93\%, 87\%, and 91\%, respectively. The results were consistent with the literature reports.

\section{Conclusion}

In summary, the diagnosis of the nature of the lung lesions, according to the initial screening of imaging, can further detect serum tumor markers for the identification of benign and malignant. Combined detection of tumor markers can significantly improve the sensitivity, specificity and accuracy of lung cancer diagnosis, and provide an effective reference value for early diagnosis and treatment.

\section{References}

[1] Luo Suxia, Chen Xiaobing, Xiao Yijun. Progress in the study of serological tumor markers related to lung cancer [J]. Cancer Progress Journal, 2005, 3(3): 252-256.

[2] Chen Feng, Li Weimin, Wang Dongmei, et al. The value of combined detection of serum tumor markers in the diagnosis of lung cancer [J]. Journal of Sichuan University (Medical Science Edition), 2008, 39(5): 832-835.

[3] Li Qunying. Value of combined tumor markers in the diagnosis of lung cancer [J]. Chinese Health Nutrition, 2013, 35(4): 211.

[4] Zhou Chao, Hu Bin, Yu Jiangyue, et al. The value of serum tumor marker detection in the diagnosis and treatment of lung cancer [J]. Chinese Journal of Practical Diagnosis and Therapy, 2011(3): 245-247.

[5] Jin Jiazhi. Value analysis of combined detection of serum tumor markers in the diagnosis of lung cancer [J]. Chinese and foreign medical care, 2013, 26(5):188-189 